\title{
Dependence of Halo Properties on Interaction History, Environment and Cosmology
}

\author{
Jeffrey P. Gardner \\ University of Washington, Department of Astronomy, Seattle, WA 98195
}

\begin{abstract}
I present results from numerical N-body simulations regarding the effect of merging events on the angular momentum distribution of galactic halos as well as a comparison of halo growth in semi-analytic vs. N-body methods. A total of six simulations are used spanning 3 cosmologies: a standard flat $\Omega_{0}=1$ model, an open $\Omega_{0}=0.3$ model and a "tilted" flat $\Omega_{0}=1$ model with spectral index $n=0.8$. In each model, one run was conducted using a spatially uniform grid of and one using a refined grid in a large void. In all three models and all environments tested, the mean angular momentum of merger remnants (halo interaction products with mass ratios 3:1 or less) is greater than non-merger remnants. Furthermore, the dispersion in the merger-remnant angular momentum distribution is smaller than the dispersion of the non-merger distribution. The interpretation most consistent with the data is that the orbital angular momentum of the interactors is important in establishing the final angular momentum of the merger product, although this conclusion is at odds with previous studies. I give the proper angular momentum distribution that should be used to describe merger remnants.

I trace the most massive progenitor of $L_{*}$ galactic-mass halos from redshift $z=0$ back to $z=5$ in the uniform grid simulations and for $10^{11} M_{\odot}$ halos in void regions. Semi-analytic mass histories match reasonably well for the latter sample. However, I find that for halos of mass $10^{12} \lesssim M \lesssim 10^{14} M_{\odot}$, the semi-analytic method underestimates the mass of progenitors and hence the formation redshift of halos in simulations. These results are independent of $\delta_{c}$. I conclude that the tested current semi-analytic implementation of halo growth and interaction does not reproduce absolute masses of progenitors or formation times above $10^{12} M_{\odot}$.
\end{abstract}

\section{Introduction}

The majority of spiral galaxies do not exist in isolation, but rather in an environment which sustains repeated close encounters and merging processes with both small and large companions Schwarzkop \& Dettmar 2000). Increasing evidence links disturbed or lopsided features of these galaxies to recent dynamical interactions. HST observations at high redshift have shown a preponderance of "train wreck" morphologies arising from major interactions. More recently, minor mergers have begun to be linked to $m=1$ mode disturbances as observed in the Fourier azimuthal decomposition of the galaxy's surface brightness, generally denoted as "lopsidedness" (Rudnick, Rix, \& Kennicut 2000; Rudnick \& Rix 1998). Specifically, studies by Richter \& Sancisi (1994) have estimated that about half of late-type spirals exhibit significantly lopsided HI profiles, and half of these are field galaxies removed from cluster environments. Furthermore, Zaritsky \& Rix find that $20 \%$ of all late-type spirals are significantly lopsided at optical and near-infrared wavelengths, yet have no obvious interactors. Rudnick \& Rix (1998) find that one fifth of all disk galaxies, regardless of Hubble type, have azimuthal asymmetries in their stellar mass distribution. They also show that this observed asymmetry is in fact due to the underlying mass distribution and not dust obscuration or other non-dynamical effects. Dynamical profiles of this nature have been shown to be 
consistent with the accretion of a satellite galaxy of a larger system (Levine \& Sparke 1998; Schwarzkopt $\&$ Dettmar 2000; and references therein). Consequently, it is probable that a substantial fraction of disk galaxies are experiencing interaction due to minor mergers.

Field spheroidals also have a number of properties such as shells, counterrotating inner disk, and bluer colors, which are believed to also be associated with recent merger events (de Carvalho \& Djorgovski 1992; Rose et al. 1994; Longhetti et al. 1998; Abraham, et al. 1999). Governato et al. (1999) show that a substantial fraction of the field elliptical population could be the result of major merging activity.

Hence, even in the field, observational evidence exists to support the hierarchical clustering scenario (HCS, see Peebles 1993) wherein larger objects are formed through the progressive assemblage of smaller mass collapsed structures. In short, the galaxy formation and evolution process is ubiquitously influenced by interactions. Therefore, to fully understand galactic formation and evolution and their consequences (both observational and theoretical), one must understand link between galaxy properties and interaction history, and how both of these may be modified by environmental effects.

The Press \& Schechter formalism $(1974$, PS) is at present the best analytical model to describe the hierarchical clustering scenario. The PS theory allows one to derive, in a relatively simple and straightforward way, the mass distribution of collapsed objects and its evolution with time in different FRW universes.

Despite its simple derivation, which relies uniquely on the linear theory, the PS formula captures the major feature of the hierarchical evolution as shown in the good fit of the PS mass function to N-body simulations Gardner et al. 1997; Lacey \& Cole 1994; Borgani et al. 1999 and references therein). Recently it has become possible to pinpoint quantitative differences between this picture and the more complex $\mathrm{N}$-body description. At cluster-sized masses, it is possible to match PS and simulated mass functions reasonably well Governato et al. 1999; Borgani et al. 1999), albeit with some tuning. However, at lower masses, evidence suggests that PS may provide a systematic overestimate of the halo number density (Bryan \& Norman 1998; Gross et al. 1998; Tormen 1998).

The PS formalism can be used to construct fast monte carlo algorithms which follow the evolution of halos (Lacey \& Cole 1993; Baugh et al. 1998 and references therein). Semi-analytic (SAM) merger trees using this "extended" PS methodology can be done with far less expenditure of resources than N-body simulations. This formalism has been used to predict a wide range of galaxy properties such as colors, morphology, merging history, and formation time (Baugh et al. 1996 and references therein). It has been used successfully to predict the same qualitative behavior found in N-body simulations. For instance, both SAM studies and N-body simulations find that the typical redshift of last major interaction of field ellipticals is lower than the cluster elliptical population (Governato et al. 1998; Baugh et al. 1996). Furthermore, SAM results also generally reproduce observed behavior such as the morphology-density relation, color-magnitude diagrams, the Butcher-Oemler effect, and the luminosity evolution of color-selected galaxy samples.

Given the great utility of SAM methods, it is crucial to understand how the SAM formalism relates to N-body simulations. Somerville et al. (1998) compare the extended Press-Schechter model and find that discrepancies as high as 50\% can exist between SAM and N-body numbers and progenitor masses, as well as the progenitor number-mass distributions and halo mass functions. On the other hand, they find that a number of more relative quantities, such as the ratio of progenitor masses, are reproduced fairly well. Tormen (1998) also finds that SAMs underpredict the number of high-mass progenitors of cluster members at high redshift and concludes that caution should be exercised when modeling clustering properties of halo progenitors. One possibility for this discrepancy might be non-spherical collapse, but Tormen measures the 
infall pattern for clusters in the sample and does not find any systematic difference between high-mass and low-mass clusters. Since the SAM vs. N-body discrepancy is not as great for low-mass clusters, it is unlikely that non-spherical collapse is responsible.

SAMs provide a representation of halos over a cosmologically representative range of environment. How applicable are SAMs in environmental extremes? Lemson \& Kauffmann (1999) use N-body simulations to examine a number of halo parameters as function of environment. They find environmental dependence only for the mass distribution of halos, with angular momentum, concentration parameter, and formation time remaining unaffected. Consequently, one would expect SAMs to maintain an as accurate a representation of environmental extremes as it does more representative regimes. In this work, I compare SAM output to $\mathrm{N}$-body simulations in both a representative volume and an extremely underdense region.

One quantity which cannot be derived from semi-analytic modeling is the angular momentum properties of a halo. Workers who study structure within galactic halos may derive halo mass and interaction histories from the extended PS formalism, but spin parameters are typically assigned from the prescriptions found by previous N-body work. For example, Dalcanton, Spergel, \& Summers (1997) use PS in combination with the spin parameter distribution of Warren et al. (1992) in order to study the distributions of surface brightness and scale length in disk galaxies. Van den Bosch (1998) uses this same distribution to examine the formation of disks and bulges within dark matter halos. Mo, Mao \& White (1998) examine the formation of disk galaxies by incorporating an a priori distribution of dark matter halo angular momentum. Any semi-analytic investigation of galaxy evolution and interaction (so-called "merger tree" studies; e.g. Somerville \& Primack 1999) must also assign spin parameters in this manner. When a halo experiences a major merging event, a new spin parameter can be assigned. However, should it be taken from the same general distribution found in N-body simulations or are the angular momenta of merger remnants systematically different from the quiescent population?

This paper is divided into two major thrusts. In the first, I examine the distribution of halo spin parameters in the overall halo population as well as merger remnants. I do this for several cosmologies, and for a large cosmologically representative volume ("uniform volume") as well as a large, underdense void. In the second thrust, I compare semi-analytic halo progenitor masses and halo formation times to N-body simulations. I examine this relation in 3 uniform volumes and two voids, which collectively sample 3 different cosmologies.

\section{Methods}

\subsection{The PS formalism}

The standard PS formula predicts the number density $N(M, z) d M$ of high contrast structure of a given mass $M$ at a given epoch. The counting of such structures is based on the hypothesis that the overdense regions in the initial density field filtered on the scale of interest, will eventually collapse to form high contrast structure. Then to obtain the fraction of the mass collapsed on a certain scale, it needs to single out volumes of the filtered density field, where the (linearly) evolved density contrast $\delta$ exceeds a nominal threshold $\delta_{c}(z)$ depending on the epoch, which guarantees that the associated volume is collapsed into a virialized object. The PS formula states:

$$
N(M, z)=\sqrt{\frac{2}{\pi}} \rho a \frac{b \delta_{c}(z)}{\sigma} M^{-2} e^{-b^{2} \delta_{c}(z)^{2} / 2 \sigma^{2}},
$$


where $\sigma^{2}(M)$ is the mass variance of the perturbation field on the scale $M$, with the normalization $b^{-1}$ which is the rms value of the linear fluctuation in the mass distribution on scales of $8 h^{-1} \mathrm{Mpc}$, the conventional way to normalize the linear spectrum. The field is taken to be Gaussian, and then the variance $S=\sigma^{2}(M)$ completely describes the density perturbation field. The threshold $\delta_{c}(z)$ is typically normalized to the canonical value $\delta_{c}(0)=1.68$, referring to the collapse of homogeneous spherical volumes, which is clearly an oversimplification; this value is used throughout this paper for simplicity, keeping in mind that the relevant parameter is the product $b \delta_{c}(0)$. The threshold $\delta_{c}(z)$ is proportional to the linear growth factor $D(t)$ (see e.g. Peebles 1993) and contains the dependence from the specific FRW model. The overall normalization is such that at any $z$ all the available mass density $\rho$ resides in collapsed objects. For a detailed derivation and the analysis of the underlying statistics, see Bond et al (1991).

Paulo Tozzi graciously furnished the author with a copy of his code, based on the methods in Lacey \& Cole (1993) which is in turn based on the "excursion set" formalism developed by Bond et al. (1991). In this method, the linear density field is smoothed at progressively larger mass scales and the mass of the halo containing a given particle at time $t_{1}$ is equal to the mass $M_{1}$ of the largest sphere which in which the average overdensity $\delta_{c}\left(t_{1}\right)$ exceeds the collapse theshold. This ensures that a halos of mass $M$ has not yet been subsumed by a larger mass halo. $\delta_{c}(t)$, of course, changes with time and thus at later times, our halo of mass $M_{1}$ and time $t_{1}$ may ultimately become part of a larger collapsed structure $M_{2}$ which does not reach critical density until time $t_{2}$. By examining the growth of linear modes in the same region, one can statistically construct halo growth and interaction histories. Tozzi's method differs from that of Lacey \& Cole (1993) in the specifics of how it calculates the relationship between mass $\left(M_{1}, M_{2}, \ldots\right)$ and collapse threshold $\left(\delta_{c}\left(t_{1}\right), \delta_{c}\left(t_{2}\right), \ldots\right)$ but yields essentially the same results (Tozzi, private communication). The method is described in detail by Goverato \& Tozzi (2000).

\subsection{Simulations}

I present 6 simulations which are evolved from 3 cosmological models: a critical universe (SCDM) $\left(\Omega_{0}=1, h \equiv H_{0} / 100 \mathrm{~km} \mathrm{~s}^{-1} \mathrm{Mpc}^{-1}=0.5, \sigma_{8}=0.7\right)$, an open $(\mathrm{OCDM})$ universe $\left(\Omega_{0}=0.3, h=0.75\right.$, $\left.\sigma_{8}=1\right)$ and a tilted critical model (TCDM) with $\mathrm{h}=0.5, \sigma_{8}=0.6$, a primordial index $\mathrm{n}=0.8$ and a gamma factor $\Gamma=0.37$. The normalizations were chosen to match the observed cluster abundance. The simulated volume was $100 \mathrm{Mpc}$ on a side (h already included) in all three runs. The parameters of the TCDM model have been chosen to satisfy both the cluster abundance and the COBE normalization at very large scales (Cavaliere, Menci, \& Tozzi 1998). This model differs from the SCDM in having less power and a steeper power spectrum at scales under $8 \mathrm{~h}^{-1} \mathrm{Mpc}$ in comparison with the other two models. Each simulation was performed using PKDGRAV (Stadel \& Quinn, in preparation) a parallel N-body treecode supporting periodic boundary conditions. One simulation in each cosmology (the "uniform volume") was run beginning from a spatially uniform grid of $144^{3}(\sim 3$ million) equal-mass particles with spline softening set to $60 \mathrm{kpc}$, allowing us to resolve individual halos with present-day circular velocities $V_{c}$ as low as $130 \mathrm{~km} \mathrm{~s}^{-1}$ with 100 particles in OCDM and $170 \mathrm{~km} \mathrm{~s}^{-1}$ with 100 particles in SCDM and TCDM. The opening angles of the SCDM and OCDM simulations were $\theta=0.5$ for redshifts $z>2$ and $\theta=0.7$ thereafter. TCDM was run with $\theta=0.5$ at all redshifts. The remaining three simulations, one in each cosmology, were of a large void approximately $40 \mathrm{Mpc}$ in diameter, and were performed using the hierarchical grids technique in which the void region is represented with large numbers of low-mass particles, while the surrounding environment is composed of small numbers of high-mass particles. This allows much greater mass resolution to be achieved while ensuring that cosmological context is maintained. I used approximately 5 million particles in each 
void simulation, with an effective resolution of $432^{3}$ across the $100 \mathrm{Mpc}$ box. Particles were spline-softened to $6.67 \mathrm{kpc}$, being $8.6 \times 10^{8} \mathrm{M}_{\odot}$ in mass in critical models and $5.8 \times 10^{8}$ in OCDM. $\theta$ was set to 0.6 for $z>2$ and 0.8 thereafter. All six simulations were evolved using between 500 and 1000 major time steps in which the forces on all particles were calculated. Each major time step is divided into a number of smaller substeps, the exact number of which is adaptively chosen by PKDGRAV such that all particles are moved on steps consonant with their dynamical times. For convenience, I shall adopt the notation of SCDMu, OCDMu and TCDMu for the uniform volumes and SCDMv, OCDMv and TCDMv for the voids.

I identify halos using the classic friends-of-friends (FOF) method (Davis et al. 1985) using a linking length that corresponds to the mean interparticle separation at the density contour defining the virial radius of an isothermal sphere: $b=\left(n \rho_{\text {vir }}(z) / \bar{\rho}(z) / 3.0\right)^{-1 / 3}$ where $n$ is the particle number density and $\rho_{\text {vir }}(z)$ is the virial density which depends on cosmology and is given in Kitayama \& Suto (1996).

\subsection{Halo Mass Histories}

One can perform two possible kinds of tests of the Press-Schechter formula on N-body simulations. In the first, one assumes as input the spectral parameter $P(k)$ and $b$, and compares the mass distribution at a given epoch. This essentially decides whether the PS formula correctly states the shapes and the normalization of the mass distribution of collapsed object. Work has been done comparing the PS mass function to N-body simulations (Lacey \& Cole 1994, Borgani et al. 1999, Governato et al. 1999, Bryan \& Norman 1998, Gross et al. 1998, Tormen 1998), and I do not use this method in my analysis.

I focus in the another test which is independent of the shape of the mass function and hence on the particular choice for the parameter as $\delta_{c}$. It consists in normalizing the PS mass function to the N-body mass function to a given epoch, and measuring the two mass distributions at precedent epochs. The PS mass formula behaves like a self-similar function plus a density evolution factor in front, and the scaling relation with redshift directly relies on the hierarchical clustering paradigm ( $c f$. Kaiser 1986). Thus, this is a test only for the time behavior of the hierarchical clustering scenario, i.e. for the hypothesis that the evolution of the density perturbation field can be described taking into account only the linear theory.

In the uniform volume simulations, I identified all halos at $\mathrm{z}=0$ of at least mass $M_{\text {res }}=2.318 \times 10^{12}$ $\mathrm{M}_{\odot}$, which corresponds to 100 particles in the flat models and roughly 150 in OCDM. With a mass-based cutoff, it is more straightforward to compare time evolution of the different cosmologies. I then "traced" each identified halo backwards in time, following the mass of the most massive progenitor as a function of redshift.

For the void simulations, I first identified the void region, which was the largest sphere that could be drawn in the simulation volume with mean overdensity $\bar{\delta}=-0.9$. The radii of the resultant spheres were 18.5 Mpc, $17 \mathrm{Mpc}$, and 13.5 Mpc for SCDMv, OCDMv and TCDMv voids respectively. Within each sphere, I identified all groups with at least 50 particles and traced them in a manner identical to the uniform volume counterparts. Unfortunately, the TCDM cosmology forms structure extremely late, and the void had only 12 halos in it greater than this mass cutoff. I therefore drop TCDMv from the majority of the following analysis.

I now wish to construct a semi-analytic realization which most accurately compares against the simulated results. Comparing individual halos is fruitless, given the extreme variation of mass histories even between halos of the same mass. Instead I wish to characterize the mean mass history of the sample, 
i.e. the average mass of a halo in the sample as a function of redshift. This was easily done by drawing the mass distribution of the halos to be modeled semi-analytically from the simulations themselves. Specifically, for each $M>M_{\text {res }}$ halo in a simulation, 100 SAM realizations of the mass history of that halo were constructed. Thus, I can directly compare the aggregate mass history of the sample of simulated halos to the collective mass history of the semi-analytic realizations.

Errors in both simulated and SAM samples were estimated using the bootstrap method. For each sample, 1000 identically-sized subsamples of halos were drawn from the $\mathrm{z}=0$ distribution. Then, for each subsample, the mean mass history was calculated and used in the bootstrap estimate.

\subsection{Halo Angular Momentum}

For SCDMu and OCDMu uniform volumes and for the OCDMv void, I determine the distribution of halo spin parameters $\lambda=L E^{1 / 2} / G M^{5 / 2}$ for recent merger remnants versus non-remnants. I select halos for this analysis based on particle number in the $z=0$ FOF group: 100 particles for SCDMu and OCDMu and 50 particles for OCDMv. The OCDMu particle cutoff is lower than in the section 2.3 for more favorable number statistics. To identify mergers, I use the groups determined by SKID, a halo-finding algorithm based on local density maxima. The SKID algorithm is similar to the DENMAX scheme (Gelb \& Bertschinger, 1994) as it groups particles by moving them along the density gradient to the local density maximum. The density field and density gradient is defined everywhere by smoothing each particle with a cubic spline weighting function of size determined by the distance encompassing the nearest 32 neighbors. At a given redshift, only particles with local densities greater than one-third of 177 times the critical density are "skidded" to the local density maximum. This threshold corresponds roughly to the local density at the virial radius. The final step of the process is to remove all particles that are not gravitationally bound to their parent halo. SKID was originally designed to find high contrast density structures within larger halos (see Ghigna et al. 1998 for a more complete description). For halos with no resolved internal substructure (i.e. no halos within halos) it gives results very similar to FOF. However, SKID does not suffer of the well known pathology of FOF of linking together close binary systems (Governato et al. 1997). Instead, it only combines two "clumps" of substructure when they are mutually gravitationally bound and thus is well suited for the task of identifying binary systems in the process of merging.

I denote a halo as a merger remnant if, at some time during $0<z<z_{m}$ it was classified as a single SKID group in one output, but two separate groups with a mass ratio $\leq 3: 1$ in the preceding output. $z_{m}$ was chosen to be as close to $z=0.5$ as the outputs would allow. In SCDMu this is $z_{m}=0.4$, and in both OCDM runs $z_{m}=0.5$.

I use SKID to identify halos before the merging event and FOF to identify the descendant halo at the present time. Once FOF identifies the halos, however, I determine the most bound particle in each FOF group and find the radius from that particle at which the enclosed density crosses the virial threshold $\rho_{\text {vir }}(z)$. The spherical overdensity (SO) method has the advantage of culling out pathologically shaped groups which depart substantially from the isothermal sphere model. The most common example is a "dumbbell" halo, which consists of two spherical halos that FOF links together by a thin bridge of particles spaced close to $\rho_{v i r}(z) / 3$. The total spin parameter of such a configuration is irrelevant to this study which is concerned only with cases where the angular momentum of a galaxy is linked to its parent halo. The spin parameter $\lambda$ is calculated by considering the contributions of all particles within the SO virial radius. If I use the FOF group membership rather than the SO, the angular momenta for non-pathological cases 
(about the FOF center of mass) is generally the same. Strangly-shaped groups are in the minority and hence even with their addition in the FOF sample, the the results remain qualitatively similar.

\section{Results}

\subsection{Angular Momentum of Merger Remnants}

The spin parameter distribution is typically expressed as the lognormal function

$$
p(\lambda) \mathrm{d} \lambda=\frac{1}{\sigma_{\lambda} \sqrt{2 \pi}} \exp \left(-\frac{\ln ^{2}(\lambda / \bar{\lambda})}{2 \sigma_{\lambda}^{2}}\right) \frac{\mathrm{d} \lambda}{\lambda}
$$

(e.g. van den Bosch 1998; Barnes \& Efstathiou 1987; Ryden 1998; Cole \& Lacey 1996; Warren et al. 1992). Figure 1 shows $p(\lambda) \mathrm{d} \lambda$ of simulated halos above $M_{\text {res }}$ at $\mathrm{z}=0$. The solid histograms denote the merger-remnant population and the dotted histograms are halos which have not experienced a major merger since $z=z_{m} \sim 0.5$. The smooth curves are fits of the form in equation 2 to the simulated distribution. Parameters for the fits are given in Table 1. Since the OCDM void model contains so few halos, it is easier to see the details of the distributions by viewing them in log space in Figure 1 $\mathrm{d}$, where the fitting function is a Gaussian. It should be noted that Lemson \& Kauffmann (1999) find no environmental dependence of spin parameter on environment. Hence, any differences in OCDMu vs. OCDMv arise from the fact that the simulations sample substantially different ranges in mass and not from environmental effects.

N-body results are often used to provide an angular momentum distributions for galaxies in semianalytic models (Dalcanton et al. 1997; Mo et al. 1998; van den Bosch 1998). Warren et al. (1992) find that the general distribution of halos peaks around $\bar{\lambda}=0.05$ with $\sigma_{\lambda}=0.7$, although later N-body researchers find distributions more consistent with $0.04 \lesssim \bar{\lambda} \lesssim 0.05$ and $\sigma_{\lambda} \approx 0.5$ (Cole \& Lacey 1996; Mo, Mao, \& White 1998; see also Steinmetz \& Bartelmann 1995; Catelan \& Theuns 1996). I find $\sigma_{\lambda}$ of all halos (in the uniform volumes) lower than 0.6 with the merger remnant population tending lower still, while the peak of the all three simulations presented is $\bar{\lambda} \sim 0.035$. Hence, my results for the global halo population are in concordance with previous findings.

I find that in all cases the distribution of spin parameters of merger remnants is greater than non-merger remnants. The K-S probabilities that the two sets were drawn from the same distribution are given as $P_{K S}$ in Table 1 and are quite low. The K-S test is most certain in SCDMv, given the large number of major mergers. Even though both OCDM runs contain fewer resolved merger remnants, the K-S test differentiates the merger and non-merger remnant populations at substantial confidence. To determine the robustness of the dissimilar means $\bar{\lambda}$ of distributions, I use the Wilcoxon test ( $c f$. Lupton 1993). The value $P_{W i l}$ given in Table 1 is the probability that the difference between the mean of the merger population the mean of the non-merger population could have occurred by chance. In all cases, the probability is quite high that $\bar{\lambda}$ of the two distributions are indeed significantly different. Consequently, spin parameters of halos which have experienced a recent major merger are distributed differently than halos which have not undergone a recent significant interaction.

Angular momentum has been found to be relatively insensitive to cosmology and collapse anisotropy

for halos formed by less violent collapse, with the spread in $\bar{\lambda}$ arising mainly from tidal effects (Huss, Jain, \& Steinmetz (1999)). Given the large spread in $\bar{\lambda}$ in my results, it seems tempting to conclude that on a halo-by-halo basis, tidal effects are more important than merger history in determining the ultimate angular 

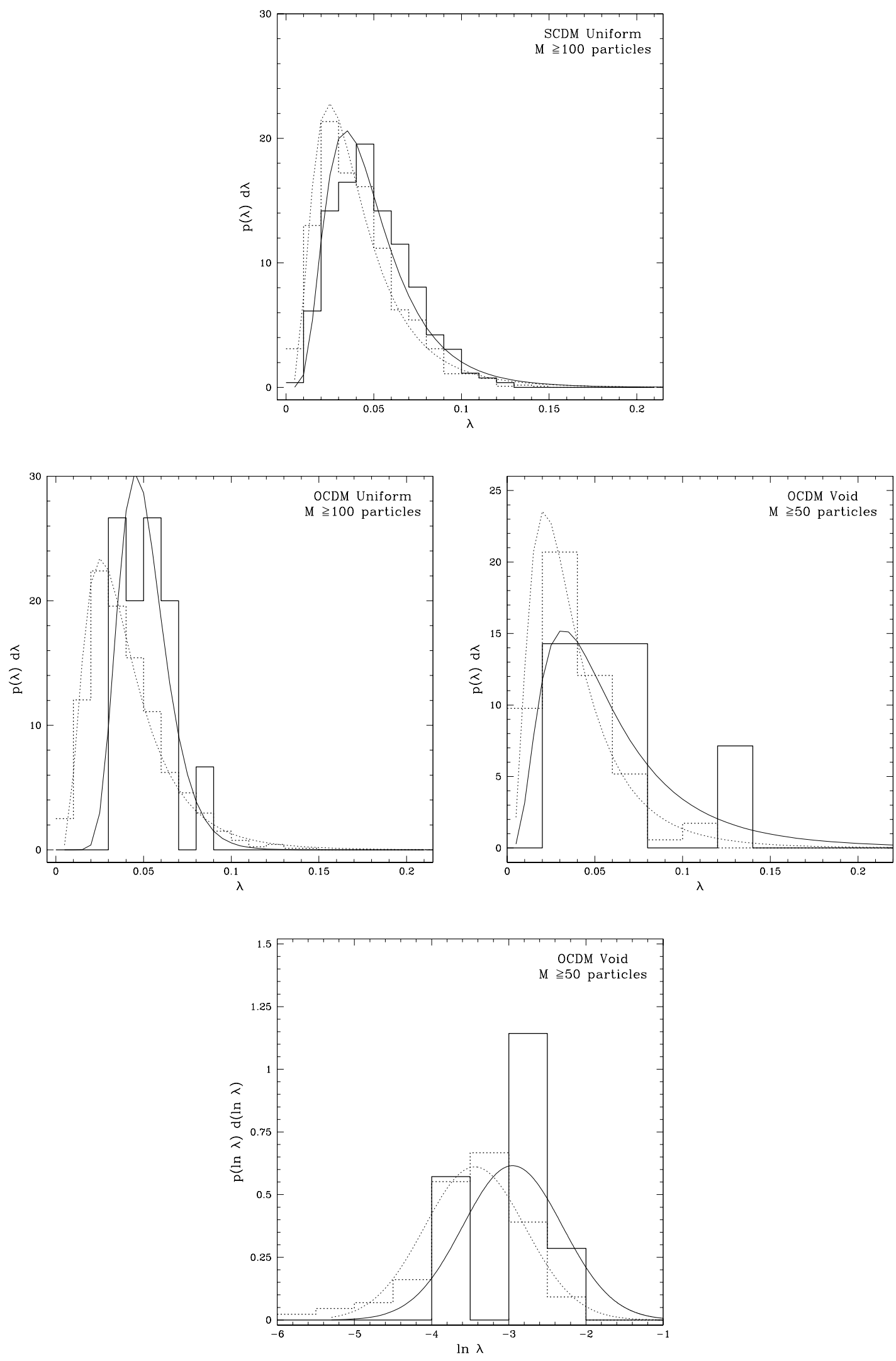

Fig. 1.- Distribution of spin parameters in flat and open uniform volumes, SCDMu and OCDMu, as well as the open-model void, OCDMv. Histograms depict the simulated data and curves show the best fit lognormal function as defined in equation 2. Dashed lines represent halos which have experienced no major merger 
momentum of a halo. Merging may be looked at simply as adding a small upward bias. However, one should note that the dispersion $\sigma_{\lambda}$ in the merger remnants is typically lower than the non-merger remnants. This can imply one of two possibilities: either the angular momentum of mergers is largely independent of tidal torques or merging adds a small bias but also selects for specific environments. In the latter, by considering mergers, we are biasing ourselves towards examining halos which are in a region that promotes merging, nurturing halos with a narrower range in $\lambda$ and a higher mean. In the former, it is the orbital angular momentum of the merger and/or the spin-alignment of the halos that determines the remnants resultant angular momentum. If only spin-alignment were important, however, then one would expect the dispersion in $\lambda$ to be larger than the non-merger distribution, as two average halos can either add their spins or have them cancel completely. Hence, either tidal torques or orbital angular momentum determine spin parameter.

These results are in contrast to Nagashima \& Gouda (1998) who, using semi-analytics (the "merging-cell model"), find that the orbital angular momentum of major mergers is not important for the remnant, and that major merging activity as a whole has little effect on the halo angular momentum distribution. The small effect that it does have is in the opposite sense of my results, with mergers having slightly lower spin parameters. Two causes come to mind which may bring about this discrepancy: the actual $\lambda$ assigned to a halo that collapses quiescently may be different than in simulations, or the orbital angular momenta of the merging systems may be more important than their studies indicate. Nagashima \& Gouda assign the angular momentum of the halo using the velocity field at the time of maximum expansion. Thus, the first instance can be examined by testing this method against halos in N-body simulations. In a very limited number of cases, the same halo exists in both OCDMu and OCDMv simulations, which are a factor of 27 different in mass resolution. For these cases, I have found the $\lambda$ of the halo to differ by up to $45 \%$. In all cases, there is at least one smaller satellite in the halo which is completely unresolved in the low-res case. It is possible that the total angular momentum of the halo is largely determined by the orbital angular momentum of clumps it consumes or with which it merges. Hence, in the low-res case, the smaller clumps go unresolved, thus altering the measures $\lambda$ of the halo. The veracity of this picture is difficult to asses. Huss et al. (1999) show evidence of the angular momentum of halos being consistent with tidal effects. However, both simulated resolutions should resolve the tidal field quite well. If on the other hand a more local effect such as accretion of clumps were to play a major role in halo spin parameter, the clumps themselves must now be resolved to such an extent that their orbital angular momentum can be reliably determined. This has the unpleasant implication that numerical resolution effects in halo spin parameter measurement need to be methodically evaluated. In any case, the tentative interpretation most consistent with my results would be that orbital angular momentum in halo-halo interactions does play a role in the final halo spin parameter. This is, however, at odds with previous semi-analytical studies and should be investigated in more detail in a future work.

How relevant is the contribution of merging to the overall spin parameter distribution? Figure 2 plots the fraction of halos in my sample with spin parameters $\lambda$ that are major merger remnants. The result is strongly dependent on cosmology, given that in open universes, merging activity at low redshift is substantially less likely than in $\Omega_{0}=1$ models (Governato et al. 1998). Consequently, the global effect of merging on halo angular momentum is simply determined by the rate of major mergers. This information is, however, of marginal utility since galaxy history or properties are rarely used to infer angular momentum. In general, the distribution $p(\lambda) d \lambda$ is employed in an a priori manner. In this case, researchers may wish to adopt a different distribution for galaxies which are merger remnants and those which are not.

The overall implication of these results is that merging activity is correlated with higher angular 
momentum. Orbital angular momentum of interaction halos would seem to be the cause most consistent my data. However, on galaxy scales, the angular momentum of ellipticals is generally much lower than spirals (Nagashima \& Gouda 1998). This is troublesome if ellipticals are to be considered merger remnants and spirals the results of long periods of quiescence. The quiescent accretion required to form a higher- $\lambda$ spiral disk would appear to take place in halos with systemically lower spin parameters. Differences in angular momenta between galaxy morphologies may be more due to formation epoch Huss, Jain, \& Steinmetz (1999) ) than merger history.

\subsection{Halo Mass Histories and Formation Times}

Here I compare the semi-analytic approximation to simulated data in differing cosmologies and environment. Figure 3 shows the mean mass histories of halos selected in accordance with section 2.3 for the uniform volumes in all three cosmologies, and the voids in SCDM and OCDM. This is essentially a measurement of the conditional mass function examined by other authors (Tormen 1998; Somerville et al. 1998; Bower 1991). It has been established in previous studies that the Press-Schechter model underestimates the number of halos relative to simulations at mass scales which lessen with increasing redshift (Somerville et al. 1998, Gross et al. 1998, Tormen 1998). Somerville et al. (1998) find that for their tilted CDM model, SAM underpredicts the masses of the most massive progenitor at redshifts $z \gtrsim 0.5$. In the flat uniform volumes, I also find that SAM systematically predicts lower-mass principle progenitors than simulations. The discrepancy in the average progenitor mass is as high as 0.1 dex in SCDM and TCDM. Interestingly enough, things are different in the OCDMu open model in contrast to the findings of Somerville et al. (1998). In this case, the semi-analytic predictions appear to be roughly in line with the simulations. Also, SAM appears to reproduce the mean mass histories of both voids reasonably well, trailing outside the $1-\sigma$ dispersion bars for SCDMv only at very high redshift. It should be noted that the void simulations sample halos of a much lower mass range resulting in higher typical formation redshifts and making a direct comparison between uniform volume and void runs somewhat hazardous. The difference in mass history between SCDMu vs. SCDMv and OCDMu vs. OCDMv is most likely caused by the difference in mass range. The important comparison is between SAM and simulated results in each regime. The masses of the halos in the void runs are somewhat lower than in the uniform volumes and thus farther from $M_{*}$ of the mass function. Since the shape of the mass function changes little in this low-mass regime, it may be possible for $10^{11} M_{\odot}$ halos to match while $10^{13} M_{\odot}$ halos do not. Somerville et al. (1998) also

\begin{tabular}{|c|c|c|c|c|c|c|c|c|c|c|c|}
\hline \multirow[b]{2}{*}{ Model } & \multicolumn{3}{|c|}{ All Halos } & \multicolumn{3}{|c|}{ Merger Remnants } & \multicolumn{3}{|c|}{ Non-Merger Remnants } & \multirow[b]{2}{*}{$P_{K S}$} & \multirow[b]{2}{*}{$P_{W i l}$} \\
\hline & $N$ & $\bar{\lambda}$ & $\sigma_{\lambda}$ & $N$ & $\bar{\lambda}$ & $\sigma_{\lambda}$ & $N$ & $\lambda$ & $\sigma_{\lambda}$ & & \\
\hline SCDMu & 1353 & 0.0366 & 0.585 & 261 & 0.0438 & 0.500 & 1092 & 0.0351 & 0.596 & $10^{-7}$ & $10^{-7}$ \\
\hline $\mathrm{OCDMu}$ & 1609 & 0.0353 & 0.568 & 15 & 0.0492 & 0.278 & 1594 & 0.0352 & 0.569 & 0.0119 & $10^{-3}$ \\
\hline OCDMv & 94 & 0.0331 & 0.660 & 7 & 0.0495 & 0.657 & 87 & 0.0320 & 0.653 & 0.320 & 0.132 \\
\hline
\end{tabular}

Table 1: Details of the halo spin parameter distribution for the sample of all halos, merger remnant halos, and non-merger remnant halos. $N$ denotes the number of halos in each distribution. The fitting parameters $\bar{\lambda}$ and $\sigma_{\lambda}$ for the lognormal distribution $p(\lambda) d \lambda$ are defined in equation 2. $P_{K S}$ is the K-S probability that the merger and non-merger populations were drawn from the same distribution. $P_{W i l}$ is the Wilcoxon probability that $\bar{\lambda}$ of the merger and non-merger populations occurred by chance. 


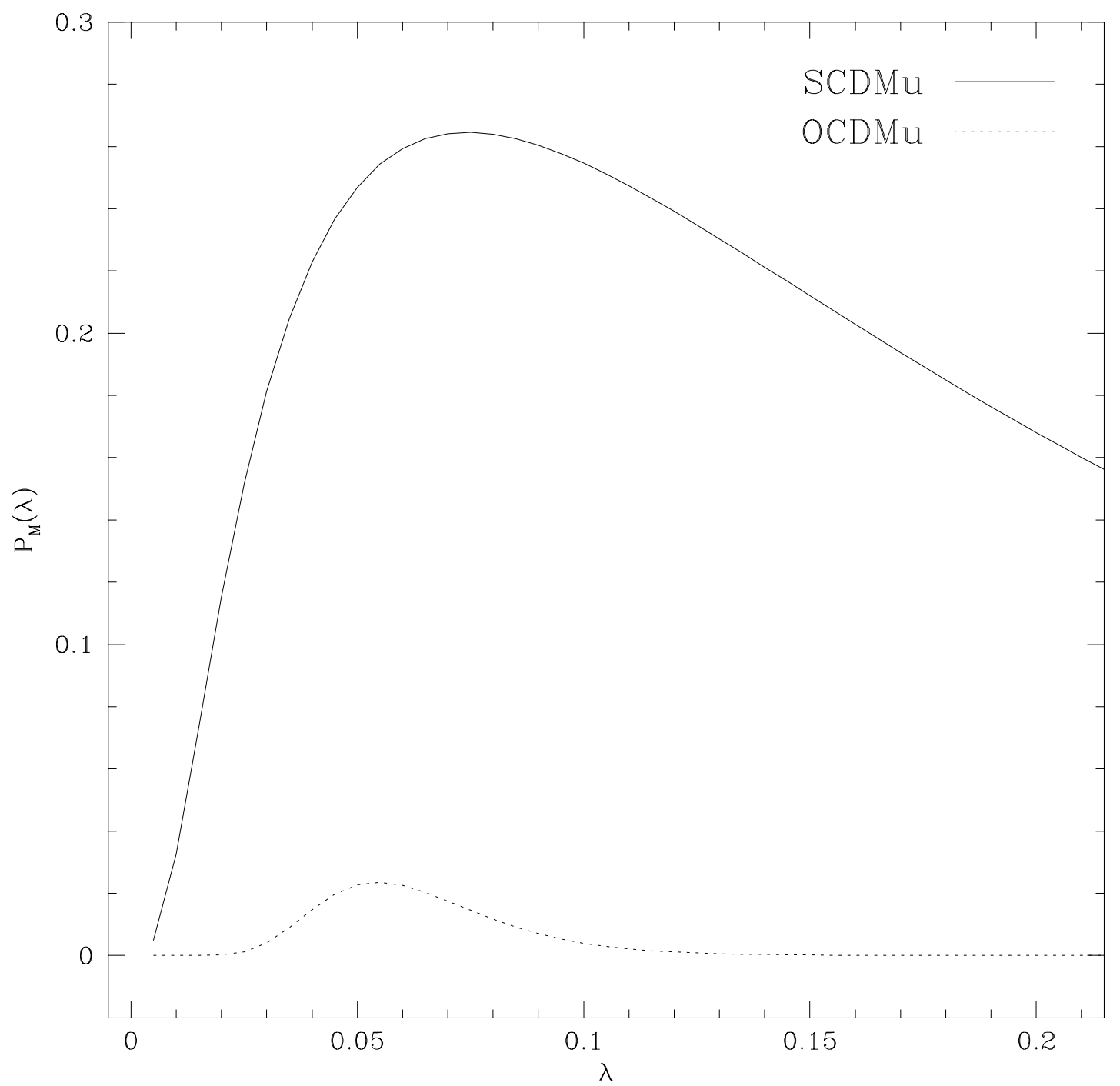

Fig. 2.- The probability $P_{M}$ that a halo with a given spin parameter $\lambda$ is a merger remnant for SCDMu (solid line) and OCDMu (dotted line). 

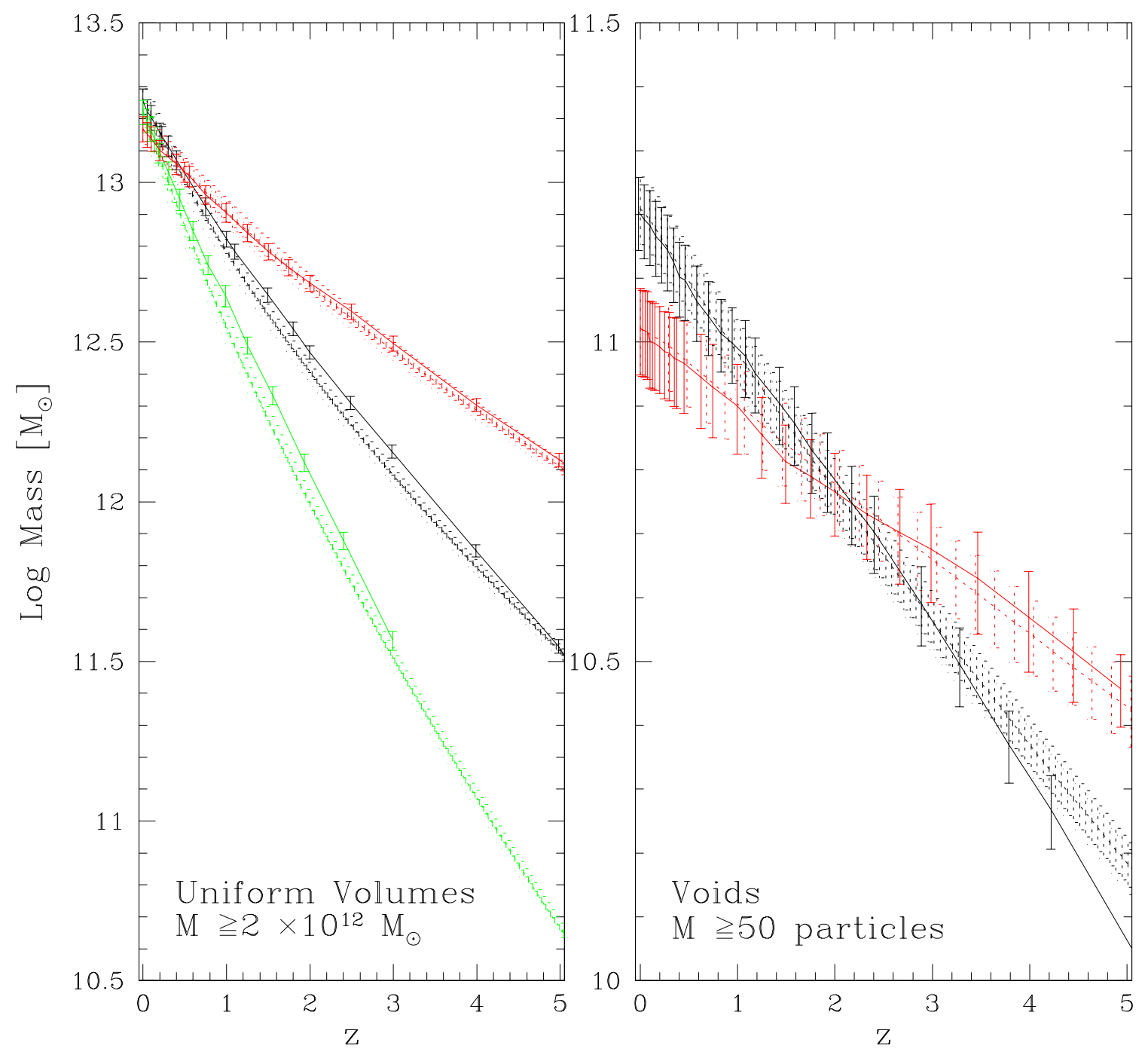

Fig. 3.- Average most massive progenitor mass vs. redshift $z$. The solid curves denote the average mass of the most massive progenitor of all halos in the simulations greater than the mass cut $M \geq 2 \times 10^{12} M_{\odot}$ (uniform volumes, left panel) or $M>50$ particles (voids, right panel). The dashed curves are the SAM realizations of the same sample of halos. The errorbars for all curves at the $1-\sigma$ deviations of 1000 bootstrap samples of the $z=0$ halo sample. In the left panel, each pair of curves (i.e. one dashed and one solid) represents a different cosmology from top to bottom view at $z=5$ : OCDMu, SCDMu, TCDMu. In the right panel, the steep pair of curves denotes the SCDMv run, and the shallower the OCDMv run. 
find that SAM progenitor masses match better for halos of mass $\sim 10^{12} M \odot$ than for halos $M>10^{13} M_{\odot}$. Hence, these results are reasonably consistent with their findings. Given the small number of halos in the void, however, these results are not conclusive and should be reexamined with a larger sample size.

The difference in results can be examined more quantitatively by comparing halo formation times. A halo is said to have formed when the mass of the most massive progenitor is $50 \%$ of the $z=0$ mass. Given the discreteness of the simulation outputs, the formation time of simulated halos can only be bounded between adjacent outputs where the halo transitions the $50 \%$ formation threshold. Figure 4 plots the formation times of halos in simulations vs. their respective SAM realizations. The points from the simulations (connected by lines) denote the number of halos formed in a bin $\Delta z$ where the bin boundaries correspond to simulation output times. In some cases multiple simulation outputs are combined into a single bin in order to increase statistics. In order to make a meaningful comparison, the formation time for each SAM realization is binned in exactly the same manner as the simulation output. The bin boundaries are given by the horizontal bars in the SAM data in Figure 4 . To determine confidence limits, I bin 1000 bootstrap samples of the SAM realization and show the regime in which $90 \%$ of the realizations fall as vertical errorbars. Consequently, for the simulation to match the SAM prediction, $90 \%$ of the simulation points should fall within the SAM errorbars. As we expect, however, from the mass history results, the SAM results are clearly different from SCDMu and TCDMu simulations at the $90 \%$ confidence level. For OCDMu, 4 out of 10 simulated points are outside the errorbars, meaning that SAM also fails to match at the $90 \%$ confidence level. In the case of the void runs, the sampling errors are too large to rule out either SAM realization, consistent with the interpretation from Figure 3. Consequently, the semi-analytic method appears to diverge notably from simulated realizations in flat models, as well as maintain a measurable difference in open models.

The difference in the results between open and flat-matter cosmologies is most likely related to the growth of fluctuations in each. Since structure grows faster in $\Omega_{0}=1$ models, the differences between SAM and simulations is most likely exaggerated with respect to low- $\Omega_{0}$.

Note again that these results are essentially independent of the value of $\delta_{c}$ chosen for Press-Schechter. The reason for this is that the mass function of halo sample at $z=0$ is set by the simulation. For each SAM realization of a simulated halo of mass $M_{i}$ at $z=0$, mass of the SAM realization is defined to be the $M_{i}$. Thus, the choice of $\delta_{c}$ merely specifies which part of the power spectrum corresponds to the mass $M_{i}$. Since the power spectrum hardly changes at galaxy scales within factors of two (the largest uncertainty in $\delta_{c}$ ), the SAM mass histories do not change, even when $\delta_{c}$ is varied substantially. For example, a shift from $\delta_{c}=1.68$ to $\delta_{c}=2.68$ in OCDMu yields a less than $1 \%$ difference in the average most massive progenitor mass, even at redshifts as high as $z=5$. Consequently, this discrepancy is not due to a tuning problem in $\delta_{c}$, nor can it be solved as such.

Governato et al. (1999) provide evidence that the best-fit $\delta_{c}$ is a function of both redshift and mass. Hence, one is effectively renormalizing the mass function at every epoch, thus changing the mapping between power spectrum and mass. If I use their fitting formula $\delta_{c}(z)=1.686\left[\left(0.7 / \sigma_{8}\right)(1+z)\right]^{-0.125}$ (valid for $\Omega_{0}=1$ ) for SCDMu, I find that this does indeed change the SAM mass history of Figure 3 b substantially. While the SAM history is lower for small redshifts, it crosses the simulated curve and eventually diverges $2 \sigma$ above the simulated histories for $z>1.4$. Governato et al. construct this relation from cluster-mass halos, well over an order of magnitude more massive than this study, so it is not surprising that using this fit does not induce SAM to match my simulated data. Still, the simulated results are bracketed with static $\delta_{c}$ producing too rapid halo growth and redshift-variant $\delta_{c}$ producing halos which grow slower than in N-body simulations. It may be possible to fine-tune a prescription for $\delta_{c}(z)$ to simulated data. As with any fine tuning of semi-analytic parameters, however, it is essential to understand the physical mechanisms 


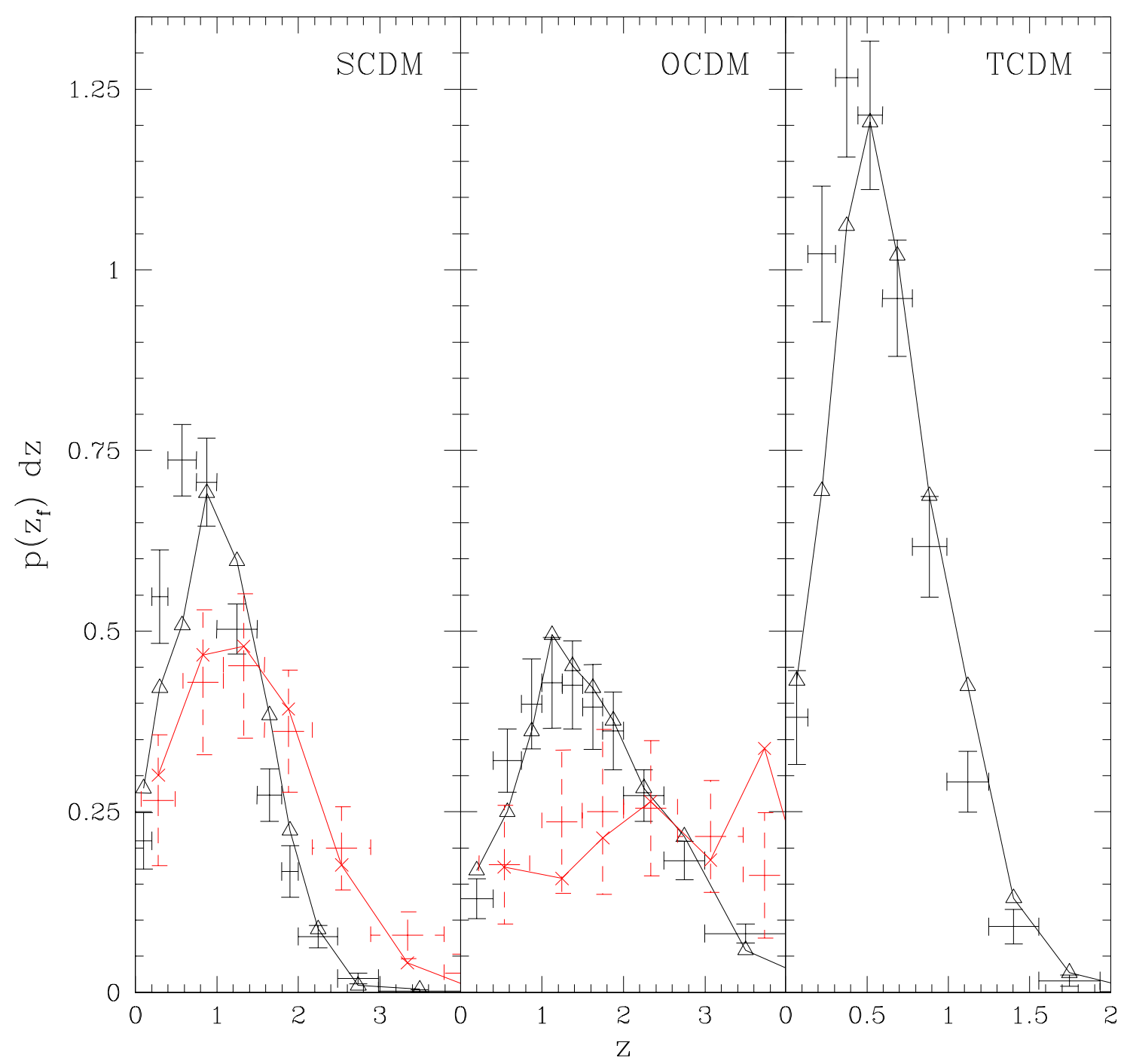

Fig. 4. - Probability distribution of formation redshift $p\left(z_{f}\right) d z$ vs. redshift $z$. In each model, the solid line and triangular points represent the uniform volume, while the solid line and starred points denote the void simulations. The SAM results are indicated by errorcrosses, with solid representing the uniform volumes and dashed representing the voids. The horizontal errorcross components denote the bin boundaries which were used for both the SAM and simulated halos. The vertical components enclose $90 \%$ of 1000 bootstrap resamplings of the SAM realizations. Thus, for SAM to match the simulated formation times the vertical errorbars must enclose 9 out of 10 points. Note that the horizontal scale for TCDM is half that of the other panels. 
underlying such parameters lest their predictive power be compromised. A study of such scope is best left to a future paper.

\section{Conclusions}

I present results from numerical N-body simulations regarding the effect of merging events on the angular momentum distribution of galactic halos as well as a comparison of halo growth in semi-analytic merger trees vs. N-body methods. In all three models tested and both in a uniform sample and in an underdense region, the distribution of halo spin parameters of merger remnants is greater than non-merger remnants. The spin parameters of the global halo population are consistent with other recent results Cole \& Lacey 1996; Mo, Mao, \& White 1998; Steinmetz \& Bartelmann 1995; Catelan \& Theuns 1996). However, the K-S probabilities of merger and non-merger samples being from the same sample are quite low, and the mean spin parameters of the two samples $\bar{\lambda}$ are significantly different. Consequently, when modeling a merger-remnant population, care should be taken to apply the correct distribution. The actual contribution of merging on the global spin parameter distribution depends on the fraction of halos that are merger-remnants and hence is greatly affected by cosmology. The overall effect of merging is to increase the mean and decrease the dispersion of the spin parameter distribution. I offer the tentative conclusion that this is consistent with the orbital angular momentum of the merging halos playing a major role in establishing the remnant's resultant net angular momentum. This interpretation is different from previous semi-analytic work, however, and should be investigated in more detail. It is also possible that angular momentum distributions could vary as a function of redshift, but I leave this for a future paper.

I trace the most massive progenitor of halos of mass $M_{i} \sim 10^{13} M_{\odot}$ at redshift $z=0$ back to $z=5$ for "uniform volumes" in all three cosmologies as well as mass $M_{i} \sim 10^{11} M_{\odot}$ halos for large voids in the $\Omega_{0}=1$ and $\Omega_{0}=0.3$ cosmologies. I find that in the uniform volumes, the semi-analytic method does not reproduce the average mass history or the distribution of formation times of the halos in the simulated sample. Because of my method of basing the PS $z=0$ mass function exclusively on simulated halos, the results are independent of $\delta_{c}$. It may be possible fine-tune SAMs to match simulated results by the use of a redshift-variant $\delta_{c}$, although such a study is beyond the scope of this paper. Sheth \& Tormen (1999) propose an improved Press-Shechter formalism along these lines which may address these issues.

My results are consistent with work by Somerville et al. (1998) and Tormen (1998). Somerville et al. find that SAMs reproduce relative properties of the progenitor halo distribution, e.g. their mass ratios, quite well. However, the absolute progenitor masses and the overall conditional mass function exhibits discrepancies of up to $50 \%$. Tormen concludes that the SAM formulation is inadequate to describe clustering in a constrained environment and may possibly be inadequate in a general context. I find that the latter is indeed true, as the conditional halo mass function in a cosmologically representative sample does not agree with simulated data. Consequently, care should be taken when comparing the raw masses of halo progenitors, or their mass relative to their eventual "children" of the present day. Formation times of

halos from SAM merger-trees are systematically biased. Furthermore, care should be taken when examining the redshift evolution of any absolute quantity which depends on the evolution of mass, such luminosity or color. Halo properties should be compared in a relative sense at each redshift and not in an absolute sense with respect to their parents or children at other times.

The author gratefully acknowledges the generosity of Paolo Tozzi for providing his semi-analytic code 
for this study. The author also thanks Fabio Governato without whose guidance and support this paper

would have been impossible, Geraint Lewis who kindly offered helpful suggestions throughout this project, and Tom Quinn who never ceases to provide useful advice and feedback. Furthermore, it is a pleasure to acknowledge frequent and useful discussions with Frank van den Bosch and Julianne Dalcanton. The author was supported by NASA Grant NGT5-50078 for the duration of this work. The simulations were performed on the Cray T3D/E at the Arctic Region Supercomputing Center.

\section{REFERENCES}

Abraham, R. G., Ellis, R. S., Fabian, A. C., Tanvir, N. R. and Glazebrook, K. 1999, MNRAS, 303, 641

Barnes, J.E., \& Efstathiou, G. 1987, ApJ, 319, 575

Baugh, C.M., Cole, S., Frenk, C.S. \& Lacey, C.G. 1998, ApJ, 498, 504

Baugh, C.M., Cole, S., \& Frenk, C.S. 1996, MNRAS, 283, 1361

Bertschinger, E. 1985 ApJS, 58, 39

Bond, J.R., Cole, S., Efstathiou, G., \& Kaiser, N., 1991, ApJ, 379, 440

Borgani, S. , Rosati, P. , Tozzi, P. \& Norman, C. 1999, ApJ, 517, 40

Bower, R.J. 1991, MNRAS, 248, 332

Bryan, G.L. \& Norman, M.L. 1998, ApJ, 495, 80

Catelan, P. \& Theuns, T. 1996, MNRAS, 282, 455

Cavaliere, A., Menci, N. \& Tozzi, P. 1998, ApJ, 501, 493

Cole, S. \& Lacey, C. 1996, MNRAS, 281, 716

Dalcanton, J.J., Spergel, D.N., \& Summers, F.J. 1997, ApJ, 482, 659

Davis, M., Efstathiou, G., Frenk, C.S., \& White, S.D.M. 1985, ApJ, 292, 371

de Carvalho, R. R., \& Djorgovski, S. 1992, ApJ, 389, 49

Gardner, J.P., Katz, N., Hernquist, L., \& Weinberg, D.H. 1997, ApJ, 484, 31.

Ghigna, S. , Moore, B. , Governato, F. , Lake, G. , Quinn, T. \& Stadel, J. 1998, MNRAS, 300, 146

Governato, F., Babul, A., Quinn, T., Tozzi, P., Baugh, C.M., Katz, N. \& Lake, G. 1999, MNRAS, 307, 949

Governato, F., Gardner, J.P., Stadel, J., Quinn, T., \& Lake, G. 1998, AJ, 117, 1651.

Governato, F. , Moore, B. , Cen, R. , Stadel, J. , Lake, G. \& Quinn, T. 1997, New Astronomy, 2, 91

Governato, F., \& Tozzi, P. 2000, in preparation

Gross, M.A.K., Somerville, R.S., Primack, J.R., Holtzman, J. \& Klypin, A. 1998, MNRAS, 301, 81

Huss, A., Jain, B. \& Steinmetz, M. 1999, MNRAS, 308, 1011 
Kaiser, N. 1986, MNRAS, 222, 323

Kauffmann, G., \& White, S.D.M., 1993, MNRAS, 261, 921

Kitayama, T. \& Suto, Y. 1996, ApJ, 469, 480

Lacey, C., \& Cole, S. 1994, MNRAS, 271, 676

Lacey, C., \& Cole, S. 1993, MNRAS, 262, 627

Lemson, G. \& Kauffmann, G. 1999, MNRAS, 302, 111

Levine, S. E., \& Sparke, L. S. 1998, ApJ, 496, L13

Longhetti, M., Rampazzo, R., Chiosi, C., \& Bressan, A. 1998, A\&AS, 130, 267

Lupton, R. 1993, Statistics in Theory and Practice, pp. 126, Princeton

Mo, H.J., Mao, S. \& White, S.D.M. 1998, MNRAS, 295, 319

Nagashima, M., \& Gouda, N. 1998, MNRAS, 301, 849

Governato, F., \& Tozzi, P. 2000, in preparation

Peebles, P.J.E. 1993, Principles of Physical Cosmology, Princeton

Press, W.H., \& Schechter, P. 1974, ApJ, 187, 425

Richter, O. \& Sancisi, R. 1994, A\&A, 290, L9

Rose, J. A., Bower, R. G., Caldwell N., Ellis R. S., Sharples, R. M., \& Teague, P. 1994, AJ, 108, 2054

Rudnick, F., \& Rix, H. 1998, AJ, 116, 1163

Rudnick, F., Rix, H., \& Kennicutt, R.C. 2000, ApJ, in preparation

Ryden, B.S. 1988, ApJ, 329, 589

Schwarzkopf, U. \& Dettmar, R. 2000, A\&AS, 144, 85

Sheth, R.K. \& Tormen, G. 1999, MNRAS, 308, 119

Somerville, R.S., Lemson, G., Kolatt, T.S., \& Dekel, A. 1998, MNRAS, submitted (astro-ph/9807277)

Somerville, R.S. \& Primack, J.R. 1999, MNRAS, 310, 1087

Steinmetz, M. \& Bartelmann, M. 1995, MNRAS, 272, 570

Tormen, G. 1998, MNRAS, 297, 648

van den Bosch, F.C. 1998, ApJ, 507, 601

Warren, M. S., Quinn, P.J., Salmon, J.K. \& Zurek, W.H. 1992, ApJ, 399, 405

White, S.D.M., Efstathiou, G., \& Frenk, C.S. 1993, MNRAS, 262, 1023

Zaritsky, D. \& Rix, H.-W. 1997, ApJ, 477, 118 